\title{
COVID-19 lockdown attack on headache emergency admissions: a multidisciplinary retrospective study
}

\author{
Laura D'Acunto $^{1}$ - Fulvio Pasquin ${ }^{1}$ - Alex Buoite Stella ${ }^{1} \cdot$ Sasha Olivo $^{1}$ - Antonio Granato ${ }^{1}$. Franco Cominotto ${ }^{2}$. \\ Paolo Manganotti ${ }^{1}$ (1)
}

Received: 6 April 2021 / Accepted: 14 August 2021 / Published online: 25 November 2021

(c) Fondazione Società Italiana di Neurologia 2021

\begin{abstract}
Background During the first COVID-19 lockdown in Italy, it was observed a reduction in emergency department (ED) attendances due to non-SARS-COV-2-related acute/chronic conditions.

Objective To analyze the impact of the COVID-19 lockdown on patients reporting headache as the principal presenting symptom on admission to the ED of the tertiary care University Hospital of Trieste over the relevant period.

Methods We retrospectively evaluated the frequency, features, and management of ED attendances for headache during the COVID-19 lockdown from 8 March to 31 May 2020, comparing it with the pre-lockdown period (January-February 2020) and the first 5 months of 2019.

Results A reduction in ED total attendances was observed in the first 5 months of 2020 compared to the same period in 2019 (21.574 and 30.364, respectively; $-29 \%$ ), in particular with respect to headache-related attendances (174 and 339 respectively; $-49 \%$ ). During the COVID-19 lockdown, it was recorded a minor reduction in the ED access rate of female patients $(p=0.03)$, while no significant variation was detected in repeaters' prevalence, diagnostic assessment, and acute treatment. The ratio of not otherwise specified, secondary, and primary headaches $(48.4 \%, 30.6 \%$, and $21.0 \%$ respectively) remained unchanged during the COVID-19 lockdown, in comparison to the control periods.

Conclusion The COVID-19 pandemic impacted the number of ED attendances for headache but not their management and setting. Despite a reduction of accesses for headache due to the pandemic emergency, the distribution of headache subtypes and the rate of repeaters did not change.
\end{abstract}

Keywords COVID-19 $\cdot$ Headache $\cdot$ Emergency department $\cdot$ Lockdown $\cdot$ Repeaters

\begin{tabular}{|c|c|}
\hline Abbreviation & \\
\hline ED & Emergency department \\
\hline SARS-COV-2 & $\begin{array}{l}\text { Severe acute respiratory syndrome coro- } \\
\text { navirus } 2\end{array}$ \\
\hline COVID-19 & Coronavirus disease 19 \\
\hline NOS & Not otherwise specified \\
\hline CT & Computed tomography \\
\hline
\end{tabular}

Paolo Manganotti

pmanganotti@units.it; neuro.strokeunit.ts@gmail.com

1 Clinical Unit of Neurology, Headache Centre, Department of Medicine, Surgery and Health Sciences, University Hospital and Health Services of Trieste - ASUGI, University of Trieste, Strada Di Fiume, 447 - 34149 Trieste, Italy

2 Emergency Department, University Hospital and Health Services of Trieste, Strada di Fiume 447, 34149 Trieste, Italy

\section{Introduction}

Italy was the first European country to experience a rapid surge of the new coronavirus SARS-CoV-2 and its associated disease (COVID-19). On February 20, 2020, the first case of COVID-19 was diagnosed in Codogno Hospital. In the following weeks, the virus had spread nationally. In an attempt to slow down SARS-CoV-2 diffusion and to reduce the impact of COVID-19 on the national healthcare system, the Italian Government imposed a nationwide lockdown on March 9, 2020. The lockdown was then prolonged until May 18,2020 , followed by the extension of several restrictions in the next 2 weeks of May, in the so-called phase two of lockdown.

During the first lockdown period, hospitals were required to establish protected wards with a view to properly managing COVID-19 patients of any severity, even for intensive and sub-intensive care needs. 
Consequently, emergency departments (EDs) were reorganized to cope with a growing number of COVID19 patients, with considerable impact on the handling of other clinical conditions. Indeed, several reports described a significant reduction from 29 to $47 \%$ of total ED admissions unrelated to COVID-19 [1-6], including with regard to conditions requiring prompt medical or surgical management [7-11]. In a single American center, it was recorded a decline in non-emergent care ED visits, in particular for patients diagnosed with hypertension, diabetes, mood and personality disorders, fluid and electrolyte disorders, abdominal pain, and, finally, headaches and migraine $(-32.5 \%)$ [12].

In the University Hospital of Trieste, located in the northeast of Italy, it was observed a decline in the number of ED attendances of several neurological conditions, such as stroke and epilepsy $[13,14]$, consistent with data collected in other centers [15-17].

Among neurological manifestations, the headache was identified as an early and common symptom of SARS$\mathrm{CoV}-2$ infection, configured as a headache attributed to systemic viral infection according to ICHD3 criteria [18]. However, its real prevalence among infected patients remained unclear, with values ranging from 6 to $71 \%$, reflecting a significant discrepancy between different studies [19]. Of a number of studies analyzing prevalence, only a few were focused on the clinical features of headache as a symptom of COVID-19. From the emerging data, no specific characteristics were described to differentiate COVID19 headache from headaches triggered by other causes, and it may present with a broad spectrum of manifestations [20-23].

Additionally, during the COVID-19 outbreak in 2020, a mild improvement of migraine features was detected in migraine patients [20, 24-26], while a worsening of migraine trend was found elsewhere [27].

While there are several studies which analyze the reorganization of headache centers during the COVID19 lockdown, none of these studies focused on changes to ED access due to headaches. The primary aim of this retrospective investigation was to study the impact of the COVID-19 lockdown on the number of patients reporting headache as the principal presenting symptom on admission to an ED. The secondary aim was to explore the clinical features, management, and setting of this subgroup of patients, compared to the pre-lockdown period (January-February 2020) and comparable periods of previous years (January-February and March-May 2019).

\section{Materials and methods}

This retrospective observational study was conducted by collecting the total number of ED attendances at the tertiary care University Hospital of Trieste during the first 5 months of 2019 and 2020. The registry was reviewed and patients with headache as the first diagnosis were identified, and headache diagnoses from ED reported on the discharge sheet were classified according to ICHD-3 criteria [18]. Demographic characteristics (age, gender), clinical features of headache on admission to the ED, anamnestic history of headache (if present), number of repeaters (patients who make at least 3 visits to the ED at least 1 week apart during a 6-month period) [28] diagnostic tests performed (lumbar puncture or CT scan), neurological, and other specialist examinations were described. ED recommendations, including an indication for hospitalization and/or to refer to the acute headache center (AHC), were also considered. Acute Headache Centre is an ambulatory service of Headache Centre of "Cattinara" University Hospital of Trieste dedicated to adult patients (age $>18$ years) with refractory to treatment migraine attack, new onset of headache, headache during pregnancy, and trigeminal autonomic cephalalgias.

The data were retrospectively collected by reviewing ED medical charts. Each patient admitted to ED provided a written and signed consent that allowed the analysis of his/her data for clinical and research purposes. All patients who attended an ED during the pandemic underwent a nasopharyngeal swab for SARS-CoV-2 upon admission. The study was conducted according to the Declaration of Helsinki and was approved by the IRB/EC of the University Hospital of Trieste.

Data analysis included all ED attendances at the hospital of Trieste for headache during the first nationwide lockdown and the following 2 weeks of the "phase two" (from 8 March to 31 May 2020), comparing it to the first 2 months of 2020 (from 1 January to 29 February 2020). Equivalent periods in 2019 ( 1 January-28 February and 8 March-31 May) were considered as matched control periods.

\section{Statistical analysis}

All analyses were performed with SPSS v.23 (IBM, Inc.). Continuous variables are presented as mean $( \pm$ standard deviation) and categorical variables as number (percentage).

January and February 2020 were considered as the "prelockdown" period, while March, April, and May 2020 were considered the "lockdown" period. Variables were compared 
between January-February and March-April-May within the same year (i.e., January-February vs March-April-May 2019 and pre-lockdown vs lockdown 2020) and between the same period in 2019 and 2020 (i.e., January-February 2019 vs pre-lockdown 2020 and March-April-May 2019 vs lockdown 2020), by using the chi-squared and Mann-Whitney $U$ tests as appropriate. $P$-values $<0.05$ were considered statistically significant.

\section{Results}

The total number of ED attendances during the first 5 months of 2019 was 30,364 , while in the same period of 2020 , it was 21,574 with a decrease of 8790 attendances $(-29 \%)$. In particular, during January-February 2019 , there were $12,146 \mathrm{ED}$ attendances, and during January-February 2020, there were 12,165 ; while in March-May 2019, there were 18,218 ED attendances; during the lockdown 2020, there were only 9409 attendances, with a decrease of $48 \%$.

During the whole considered period in 2019 and 2020, the number of patients admitted to ED due to headache was, respectively, 339 and $174(-49 \%)$. During the lockdown period, headache-related attendances were $62(0.66 \%, 5.6$ per week), that is an evident decrease compared to the prelockdown number in $2020(0.92 \%$ vs $0.66 \%, p<0.05)$ and in March-May 2019 (1.20\% vs 0.66\%, $p<0.001)$. No significant variations were detected between January-February 2019 and March-May 2019 (Table.1).
The comparison of demographic data between March-May 2019 and March-May 2020 showed a mild reduction of age $(51 \pm 19$ vs. $46 \pm 17, p 0.053)$, with a significant reduction of female ED access rate $(72.0 \%$ vs $58.0 \%$, p 0.036). No variations in demographic data were detected between lockdown and pre-lockdown.

During the lockdown, repeaters' prevalence remained unchanged with respect to pre-lockdown 2020 and to March-May 2019, while a reduction was observed by comparison to figures recorded in January-February 2019 and 2020 (40.5\% vs $25.0 \%, p$ 0.012). More in detail, 25 repeaters visited the ED from 1 January to 20 February 2020, 5 repeaters visited the ED from 21 February to 7 March 2020, and at least 17 repeaters visited the ED during the lockdown.

In January-May 2020, neurology consultancy was required with less frequency compared to $2019(p<0.01)$, while no significant variation was recorded between January-February and March-May 2020 (Table 1). The referral to other specialist consultancies was more frequent during the lockdown period compared with pre-lockdown 2020 ( $p$ 0.003), but without significant variation with respect to March-May 2019. A significant decrease of other specialist examinations was recorded during January-February 2020 compared with January-February 2019 ( $p$ 0.044). No significant variations regarding diagnostic procedures (head CT scan, lumbar puncture), acute treatment, and rate of hospitalization were detected. A downward trend of patients referred to the AHC during lockdown was observed in comparison to March-May 2019 ( $p$ 0.077).

Table 1 Emergency department's attendances for headache: demographic data, diagnostic assessment, and discharge dispositions

\begin{tabular}{|c|c|c|c|c|c|c|}
\hline & \multicolumn{3}{|c|}{ January-February } & \multicolumn{3}{|c|}{ March-April-May } \\
\hline & 2019 & 2020 & $P$ value & 2019 & 2020 & $P$ value \\
\hline Total accesses (access/week) & $\begin{array}{l}121(\mathbf{0 . 9 9 \% )} \\
\text { (15/week) }\end{array}$ & $\begin{array}{l}112(\mathbf{0 . 9 2 \%})^{\mathrm{a}} \\
(14 / \text { week })\end{array}$ & 0.545 & $\begin{array}{l}218(\mathbf{1 . 2 0} \%) \\
\text { (20/week) }\end{array}$ & $\begin{array}{l}62(\mathbf{0 . 6 6 \%})^{\mathrm{a}} \\
(6 / \text { week })\end{array}$ & $<0.001$ \\
\hline Age (y) & $51( \pm 19)$ & $47( \pm 19)$ & 0.065 & $51( \pm 19)$ & $46( \pm 17)$ & 0.053 \\
\hline Females, $n(\%)$ & $83(69.0 \%)$ & $71(63.0 \%)$ & 0.402 & $157(72.0 \%)$ & $36(58.0 \%)$ & 0.036 \\
\hline Repeaters & $49(40.5 \%)$ & $28(25 \%)$ & 0.012 & $73(33.5 \%)$ & $17(27.4 \%)$ & 0.367 \\
\hline Neurology consultancy & $76(62.8 \%)$ & $41(36.6 \%)$ & $<0.001$ & $138(63.3 \%)$ & $28(45.2 \%)$ & 0.010 \\
\hline Other specialist consultancy & $26(21.5 \%)$ & $13(11.6 \%)^{\mathrm{a}}$ & 0.044 & $40(18.3 \%)$ & $15(24.2 \%)^{\mathrm{a}}$ & 0.307 \\
\hline Head CT scan & $82(67.8 \%)$ & $63(56.2 \%)$ & 0.070 & $150(68.8 \%)$ & $40(64.5 \%)$ & 0.523 \\
\hline Lumbar puncture & $2(1.6 \%)$ & $3(2.6 \%)$ & 0.589 & $7(3.2 \%)$ & $1(1.6 \%)$ & 0.505 \\
\hline Indication to refer to $\mathrm{AHC}$ & $29(23.9 \%)$ & $23(20.5 \%)$ & 0.530 & $42(19.3 \%)$ & $6(9.7 \%)$ & 0.077 \\
\hline Hospitalizations & $8(6.6 \%)$ & $5(4.5 \%)$ & 0.476 & $20(9.2 \%)$ & $3(4.8 \%)$ & 0.273 \\
\hline
\end{tabular}

$y$, years; $n$, number; $C T$, computed tomography; $A H C$, acute headache center

${ }^{a}$ Statistical significance between January-February 2020 and March-April-May 2020 
Table 2 Headache diagnosis on discharge sheet classified according to International Classification of Headache Disorders (ICHD) 3 criteria

\begin{tabular}{|c|c|c|c|c|c|c|}
\hline \multirow[t]{2}{*}{ ICHD 3 classification } & \multicolumn{3}{|c|}{ January-February } & \multicolumn{3}{|c|}{ March-April-May } \\
\hline & 2019 & 2020 & $P$ value & 2019 & 2020 & $P$ value \\
\hline Primary headache & $40(33.0 \%)$ & $28(25.0 \%)$ & 0.608 & $70(32.1 \%)$ & $13(21.0 \%)$ & 0.245 \\
\hline Secondary headache & $33(27.3 \%)$ & $37(33.0 \%)$ & & $68(31.2 \%)$ & $19(30.6 \%)$ & \\
\hline Facial pains & $2(1.7 \%)$ & $2(1.8 \%)$ & & $3(1.4 \%)$ & $0(0.0 \%)$ & \\
\hline Not other specified headache & $46(38.0 \%)$ & $45(40.2 \%)$ & & $77(35.3 \%)$ & $30(48.4 \%)$ & \\
\hline Total & $121(100 \%)$ & $112(100 \%)$ & & $218(100 \%)$ & $62(100 \%)$ & \\
\hline
\end{tabular}

Among the 62 patients who attended ED for headache in March-May 2020, only one resulted positive to SARSCoV-2 nasopharyngeal swab, presenting with fever and breathlessness.

During the lockdown period, "not otherwise specified headache" remained the most prevalent diagnosis $(48.4 \%)$, followed by secondary headache $(30.6 \%)$, and finally primary headache $(21 \%)$. No significant variations were observed with respect to pre-lockdown levels and to the same matched control period in 2019 (Table 2).

Secondary headaches were mostly represented by headaches attributed to arterial hypertension, vascular disorders, or injury to the head. Among primary headaches, migraine represented $14.5 \%$ of the sample, while tension-type headache covered $6.5 \%$ (more detailed data are reported in Appendix Table 3). Details of diagnosis during 2019 are reported in Appendix Table 4.

\section{Discussion}

During the first Italian COVID-19 lockdown in 2020, a consistent decrease in the total number of ED attendances at Trieste ED was recorded, compared to the corresponding period in 2019. This data is in line with other studies [1-6]. Results of this study highlight that ED admissions due to headaches were also significantly impacted by the COVID-19 outbreak.

During the first 5 months of 2020, ED access for headaches decreased by $49 \%$, especially during the lockdown period, with an average of 6 accesses per week $(0.66 \%)$, compared to 14 accesses pre-lockdown in $2020(0.92 \%)$ and to 20 accesses per week during the control period in 2019 (1.20\%). This general drop of ED visitors due to headache might depend on several causes, including the restrictions imposed by the Italian Government requiring people to stay at home and, with less probability, on the diffuse fear of being infected with SARS-CoV-2 in a hospital setting. Regarding primary headaches, the reduction in ED accesses may also be partially explained by the mild improvement of migraine symptoms trends recorded during lockdown [24].

The results of this investigation show a significant decrease in the rate of female patients' access by comparison to levels recorded both pre-lockdown in 2020 and in 2019, although females remained the majority of the overall headache patients. The greater percentage decline of female patients' attendance, compared to male patients' attendance, could reflect a stronger resilience of female patients.

The handling of headache cases in the ED remained similar to pre-lockdown and previous year periods, despite the difficult challenges posed by the pandemic outbreak and the consequent necessary ED reorganization. No variations were observed regarding diagnostic procedures (head CT scan, lumbar puncture), acute treatments, and rate of hospitalization. However, it was observed a downward trend of patients referred to the AHC during the lockdown in comparison to levels recorded in March-May 2019. This could be explained on the basis that the activity of the AHC as an ambulatory service was severely reduced by the neurological department's reorganization during the lockdown.

Among all headache ED attendances, the ratio of not otherwise specified (NOS), secondary, and primary headaches (according to ICHD 3 criteria) remained unchanged during the lockdown period, in comparison to all other considered periods (Table 2). This shows how, despite a reduction of total access for headache due to the pandemic emergency, the distribution of headache subtypes did not change. NOS remained the most prevalent discharge diagnosis from ED (48.4\%), consistently with the findings in literature before the COVID-19 pandemic [29, 30]. Secondary headaches were one-third of the sample $(30.6 \%)$, mostly represented by headaches 
attributed to arterial hypertension, vascular disorders, and injury to the head $(26.2 \%, 21.1 \%$, and $21.1 \%$ respectively). Out of the pandemic period, potentially lifethreatening secondary headaches represent about $5 \%$ of all acute headaches per year [31]. As expected, a portion of people who did not refer to ED probably complained of an acute headache with serious underlying pathology. This hypothesis is supported by a global reduction in the hospitalization of severe conditions with headache as a presenting or satellite symptom (e.g., acute stroke) [17, 32]. The observed decrease of headaches secondary to other infectious diseases (not related to SARS-CoV-2 infection) during the lockdown may be explained by a reported drop of infectious diseases as a result of pandemic prevention measures (masks, social distancing, hand washing) [33, 34]. Moreover, the revised triage method in ED adopted over this period stressed other infection symptoms suggestive of COVID-19 rather than headache alone, considering them more relevant in ED admission sheets. This last hypothesis is supported by the fact that only one patient in our study population with headache as a principal reported symptom resulted positive to SARS-CoV-2 on a nasopharyngeal swab. Among primary headaches, migraine remained the prevalent diagnosis.

Analyzing the proportion of "repeaters" (migraines patients who frequently attend ED [28]), we observed a significant drop in attendance in January-February 2020 compared to January-February 2019, while during the lockdown no changes were reported compared to the other reference periods. In the last 2 years, the AHC service has progressively improved the collaboration with the ED and general practice doctors on the education of patients suffering refractory headache to rapidly address to AHC instead of ED. This could be a possible explanation of the reduction of repeaters' accesses during pre-lockdown in 2020. On the other hand, several limitations imposed in connection with the pandemic state on general practice doctors and on ambulatory services, including AHC, have impacted on two important drainage ditches for patients suffering a headache, who in case of extreme need had to go to the ED. This could explain the minor lockdown impact on this patient's subgroup.

Compared to 2019, during the first 5 months of 2020, we observed a significant reduction of requests for neurology consultancy, independently of the pandemic period. A possible explanation could be that ED physicians in the presence of benign headaches, normal neuroimaging, and substantial relief of pain after acute ED treatment did not require further neurology consultancy in ED because of the possibility to rapidly address patients to AHC. In particular, during the last year, ED physicians were trained to rapidly recognize primary and secondary headaches and therefore to alert the neurologist only in challenging cases. On the other hand, during the pandemic period, we observed an increased request for other specialist consultancies, probably for a broader investigation of patients' symptoms.

To the best of the authors' knowledge, this is the first study that analyzed all ED attendances for headache as a principal presenting symptom and how prevalence, management, and setting of this patients' population were modified during the first Italian COVID-19 lockdown in 2020. The main limitations are the monocentric nature of the study and the inability to provide measures of the prevalence of headache among COVID-19 patients who attended the ED due to respiratory distress or suspect COVID-19 as the main diagnosis.

\section{Conclusions}

The COVID-19 lockdown impacted the number of accesses of several neurological conditions in our ED, including headache; nevertheless, the COVID-19 outbreak did not impact the ED management and setting of this subgroup of patients. Despite a reduction of total access for headache due to the pandemic emergency, the distribution of NOS, secondary, and primary headaches did not change among ED attendances, reflecting a non-subverting of these categories of headache ED admissions.

This could suggest that in this context of global reduction of admissions due to headache, an equivalent portion of patients complaining of primary and secondary headaches, probably including also potentially life-threatening ones, did not refer to the ED, for the severe limitations posed by the pandemic, whereas a still high portion of repeaters continued to present to the ED due to the reduction of ambulatory services.

On this basis and in anticipation of further periods of restriction, a strengthening of headache care telemedicine conducted by headache centers or general physicians could favor social distancing, improve the diagnosis and treatment of patients with primary/benign headache, and refer to the ED patients with suspected potentially dangerous secondary headaches, in order to reduce patients flow to ED, including also the repeaters, in this epochal event as well as in the future. 


\section{Appendix}

Table 3 Focus on discharge diagnosis in 2020 classified according to International Classification of Headache Disorders (ICHD) 3 criteria

\begin{tabular}{|c|c|c|c|}
\hline \multicolumn{2}{|l|}{ Diagnosis n (\%) } & \multirow{2}{*}{$\begin{array}{c}\text { Jan-Feb } 20 \\
17(\mathbf{1 5 . 2 \% )}\end{array}$} & \multirow{2}{*}{$\frac{\text { Mar-May } 20}{9(\mathbf{1 4 . 5 \% )}}$} \\
\hline Migraine & $26(14.9 \%)$ & & \\
\hline Migraine with aura & $11(42.4 \%)$ & $10(58.8 \%)$ & $1(11.1 \%)$ \\
\hline Typical aura without migraine & $1(3.8 \%)$ & $0(0.0 \%)$ & $1(11.1 \%)$ \\
\hline Migraine without aura & $9(34.6 \%)$ & $5(29.4 \%)$ & $4(44.5 \%)$ \\
\hline Chronic Migraine & $3(11.5 \%)$ & $1(5.9 \%)$ & $2(22.2 \%)$ \\
\hline Migrainous status & $2(7.7 \%)$ & $1(5.9 \%)$ & $1(11.1 \%)$ \\
\hline Tension Type Headache & $12(6.9 \%)$ & $8(7.1 \%)$ & $4(6.5 \%)$ \\
\hline Cluster Headache & $3(1.7 \%)$ & $3(2.7 \%)$ & $\mathbf{0}(\mathbf{0 . 0 \%})$ \\
\hline Secondary Headache & $56(32.2 \%)$ & $37(33.0 \%)$ & $19(30.6 \%)$ \\
\hline Headache attributed to trauma or injury to the head and/or neck & $7(12.5 \%)$ & $3(8.1 \%)$ & $4(21.1 \%)$ \\
\hline Headache attributed to cranial and/or cervical vascular disorder & $6(10.7 \%)$ & $2(5.4 \%)$ & $4(21.1 \%)$ \\
\hline Headache attributed to non-vascular intracranial disorder & $2(3.6 \%)$ & $0(0.0 \%)$ & $2(10.5 \%)$ \\
\hline Headache attributed to a substance or its withdrawal & $5(8.9 \%)$ & $4(10.8 \%)$ & $1(5.3 \%)$ \\
\hline Headache attributed to infection & $9(16.1 \%)$ & $7(18.9 \%)$ & $2(10.5 \%)$ \\
\hline Headache attributed to disorder of homoeostasis & $22(39.3 \%)$ & $17(46.0 \%)$ & $5(26.2 \%)$ \\
\hline $\begin{array}{l}\text { Headache or facial pain attributed to disorder of the cranium, neck, eyes, } \\
\text { ears, nose, sinuses, teeth, mouth }\end{array}$ & $5(8.9 \%)$ & $4(10.8 \%)$ & $1(5.3 \%)$ \\
\hline Headache attributed to psychiatric disorder & $0(0.0 \%)$ & $0(0.0 \%)$ & $0(0.0 \%)$ \\
\hline Painful lesions of the cranial nerves and other facial pain & $2(1.2 \%)$ & $2(1.8 \%)$ & $0(0.0 \%)$ \\
\hline Not other specified Headache & $75(43.1 \%)$ & $45(40.2 \%)$ & $30(48.4 \%)$ \\
\hline Total & $174(100 \%)$ & $112(100 \%)$ & $62(100 \%)$ \\
\hline
\end{tabular}

Table 4 Focus on discharge diagnosis in 2019 classified according to International Classification of Headache Disorders (ICHD) 3 criteria

\begin{tabular}{|c|c|c|c|}
\hline Diagnosis n (\%) & & Jan-Feb 19 & Mar-May 19 \\
\hline Migraine & $78(23.0 \%)$ & $26(21.5 \%)$ & $52(23.8 \%)$ \\
\hline Migraine with aura & $27(34.6 \%)$ & $9(34.6 \%)$ & $18(34.6 \%)$ \\
\hline Typical aura without migraine & $1(1.3 \%)$ & $0(0.0 \%)$ & $1(1.9 \%)$ \\
\hline Migraine without aura & $26(33.3 \%)$ & $10(38.5 \%)$ & $16(30.8 \%)$ \\
\hline Chronic Migraine & $11(14.1 \%)$ & $2(7.7 \%)$ & $9(17.3 \%)$ \\
\hline Migrainous status & $13(16.7 \%)$ & $5(19.2 \%)$ & $8(15.4 \%)$ \\
\hline Tension Type Headache & $31(9.1 \%)$ & $14(11.6 \%)$ & $17(7.8 \%)$ \\
\hline Cluster Headache & $1(0.3 \%)$ & $\mathbf{0}(\mathbf{0 . 0} \%)$ & $1(0.5 \%)$ \\
\hline Secondary Headache & $101(29.8 \%)$ & $33(27.3 \%)$ & $68(31.2 \%)$ \\
\hline Headache attributed to trauma or injury to the head and/or neck & $19(18.8 \%)$ & $7(21.2 \%)$ & $12(17.6 \%)$ \\
\hline Headache attributed to cranial and/or cervical vascular disorder & $10(9.9 \%)$ & $3(9.1 \%)$ & $7(10.3 \%)$ \\
\hline Headache attributed to non-vascular intracranial disorder & $12(11.9 \%)$ & $3(9.1 \%)$ & $9(13.2 \%)$ \\
\hline Headache attributed to a substance or its withdrawal & $2(2.0 \%)$ & $0(0.0 \%)$ & $2(3.0 \%)$ \\
\hline Headache attributed to infection & $19(18.8 \%)$ & $5(15.1 \%)$ & $14(20,6 \%)$ \\
\hline Headache attributed to disorder of homoeostasis & $31(30.7 \%)$ & $12(36.4 \%)$ & $19(27.9 \%)$ \\
\hline $\begin{array}{l}\text { Headache or facial pain attributed to disorder of the cranium, neck, eyes, ears, nose, } \\
\text { sinuses, teeth, mouth }\end{array}$ & $3(3.0 \%)$ & $1(3.0 \%)$ & $2(3.0 \%)$ \\
\hline Headache attributed to psychiatric disorder & $5(4.9 \%)$ & $2(6.1 \%)$ & $3(4.4 \%)$ \\
\hline Painful lesions of the cranial nerves and other facial pain & $5(1.5 \%)$ & $2(1.6 \%)$ & $3(1.4 \%)$ \\
\hline Not other specified Headache & $123(36.3 \%)$ & $46(38.0 \%)$ & $77(35.3 \%)$ \\
\hline Total & $339(100 \%)$ & $121(100 \%)$ & $218(100 \%)$ \\
\hline
\end{tabular}


Acknowledgements The authors would like to thank Victor Harlow for English proofreading.

\section{Declarations}

Conflict of interest The authors declare no competing interests.

\section{Ethical approval None.}

Informed consent Each patient admitted to ED provided a written and signed consent that allowed the analysis of his/her data for clinical and research purposes. This study was approved by the IRB/EC of the University Hospital of Trieste.

\section{References}

1. Fahrner R, Bähler S, Lindner G (2021) COVID-19 lock-down significantly reduced number of surgical presentations in an emergency department [published online ahead of print, 2021 Jan 28]. Wien Klin Wochenschr 1-4.

2. Ojetti V, Covino M, Brigida M et al (2020) Non-COVID Diseases during the pandemic: where have all other emergencies gone? Medicina (Kaunas) 56(10):512

3. Montagnon R, Rouffilange L, Agard G, Benner P, Cazes N, Renard A (2021) Impact of the COVID-19 pandemic on emergency department use: focus on patients requiring urgent revascularization. J Emerg Med 60(2):229-236

4. Jeffery MM, D'Onofrio G, Paek H et al (2020) Trends in emergency department visits and hospital admissions in health care systems in 5 states in the first months of the COVID-19 pandemic in the US. JAMA Intern Med 180(10):1328-1333

5. Bellan M, Gavelli F, Hayden E, et al (2020) Pattern of emergency department referral during the Covid-19 outbreak in Italy [published online ahead of print, 2020 Jun 16]. Panminerva Med https://doi.org/10.23736/S0031-0808.20.04000-8.

6. Morello F, Bima P, Ferreri E, et al (2021) After the first wave and beyond lockdown: long-lasting changes in emergency department visit number, characteristics, diagnoses, and hospital admissions. Intern Emerg Med

7. Neufeld MY, Bauerle W, Eriksson E, et al (2020) Where did the patients go? Changes in acute appendicitis presentation and severity of illness during the coronavirus disease 2019 pandemic: a retrospective cohort study [published online ahead of print, 2020 Dec 4]. Surgery S0039-6060(20)30745-5.

8. Garcia S, Stanberry L, Schmidt C, et al (2020) Impact of COVID19 pandemic on STEMI care: an expanded analysis from the United States [published online ahead of print, 2020 Aug 7]. Catheter Cardiovasc Interv https://doi.org/10.1002/ccd.29154

9. Vecchio S, Fileti L, Reggi A, Moschini C, Lorenzetti S, Rubboli A (2020) Impact of the COVID-19 pandemic on admissions for acute coronary syndrome: review of the literature and singlecenter experience. G Ital Cardiol (Rome) 21(7):502-508

10. Madanelo M, Ferreira C, Nunes-Carneiro D et al (2020) The impact of the coronavirus disease 2019 pandemic on the utilisation of emergency urological services. BJU Int 126(2):256-258

11. Boschetti GA, Gregorio SD, Vera JMM, et al (2021) COVID19 impact on vascular surgery practice: experience from an Italian university regional hub center for vascular pathology [published online ahead of print, 2021 Feb 4]. Ann Vasc Surg S0890-5096(21)00120-5

12. Giannouchos TV, Biskupiak J, Moss MJ, Brixner D, Andreyeva E, Ukert B (2021) Trends in outpatient emergency department visits during the COVID-19 pandemic at a large, urban, academic hospital system. Am J Emerg Med 40:20-26

13. Naccarato M, Scali I, Olivo S et al (2020) Has COVID-19 played an unexpected "stroke" on the chain of survival? J Neurol Sci. 414:116889

14. Cheli M, Dinoto A, Olivo S et al (2020) SARS-CoV-2 pandemic and epilepsy: the impact on emergency department attendances for seizures. Seizure 82:23-26

15. Granata T, Bisulli F, Arzimanoglou A, Rocamora R (2020) Did the COVID-19 pandemic silence the needs of people with epilepsy? Epileptic Disord 22(4):439-442

16. Frisullo G, Brunetti V, Di Iorio R et al (2020) Effect of lockdown on the management of ischemic stroke: an Italian experience from a COVID hospital. Neurol Sci 41(9):2309-2313

17. Jasne AS, Chojecka P, Maran I et al (2020) Stroke code presentations, interventions, and outcomes before and during the COVID19 pandemic. Stroke 51(9):2664-2673

18. Headache Classification Committee of the International Headache Society (IHS) (2018) The International Classification of Headache Disorders, 3rd edition. Cephalalgia 38(1):1-211

19. Tolebeyan AS, Zhang N, Cooper V, Kuruvilla DE (2020) Headache in patients with severe acute respiratory syndrome coronavirus 2 infection: a narrative review. Headache 60(10):2131-2138. https://doi.org/10.1111/head.13980

20. Dos Anjos de Paula RC, de Maria Frota Vasconcelos T, da Costa FBS, et al (2021) Characterization of headache in COVID-19: a retrospective multicenter study [published online ahead of print, 2021 May 25]. Mol Neurobiol 1-8

21. Rocha-Filho PAS, Magalhães JE (2020) Headache associated with COVID-19: Frequency, characteristics and association with anosmia and ageusia. Cephalalgia 40(13):1443-1451

22 Membrilla JA, de Lorenzo Í, Sastre M, Díaz de Terán J (2020) Headache as a cardinal symptom of coronavirus disease 2019: a cross-sectional study. Headache. 60(10):2176-2191

23. Coppola A, Tonini MC, Baratelli E, Barillà C, Bassani R, Gonano EF, et al (2020) Headache in a group of SARS-COVID-19 patients: an observational prospectical study. Neurol Sci 1-2. https://doi.org/10.1007/s10072-020-04676-z

24. Delussi M, Gentile E, Coppola G et al (2020) Investigating the effects of COVID-19 quarantine in migraine: an observational cross-sectional study from the Italian National Headache Registry (RICe). Front Neurol 11:597881

25. Parodi IC, Poeta MG, Assini A, Schirinzi E, Del Sette P (2020) Impact of quarantine due to COVID infection on migraine: a survey in Genova. Italy Neurol Sci 41(8):2025-2027

26. Verhagen IE, van Casteren DS, Lentsch SV, Terwindt GM (2021) Effect of lockdown during COVID-19 on migraine: a longitudinal cohort study [published online ahead of print, 2021 Jan 11]. Cephalalgia 333102420981739. https://doi.org/10.1177/0333102420 981739.

27. Al-Hashel JY, Ismail II (2020) Impact of coronavirus disease 2019 (COVID-19) pandemic on patients with migraine: a web-based survey study. J Headache Pain 21(1):115. Published 2020 Sep 24

28. Villani V, Di Stani F, Vanacore N, Scattoni L, Cerbo R, Bruti G (2010) The, "repeater" phenomenon in migraine patients: a clinical and psychometric study. Headache 50(3):348-356

29. Sahai-Srivastava S, Desai P, Zheng L (2008) Analysis of headache management in a busy emergency room in the United States. Headache 48(6):931-938

30. Relja G, Granato A, Capozzoli F et al (2005) Nontraumatic headache in the emergency department: a survey in the province of Trieste. J Headache Pain 6(4):298-300

31. Filler L, Akhter M, Nimlos P (2019) Evaluation and management of the emergency department headache. Semin Neurol 39(1):20-26 
32. De Bonis P, Cavallo MA, Sturiale CL, et al (2021) Incidence of hemorrhagic cerebrovascular disease due to vascular malformations during the COVID-19 national quarantine in Italy [published online ahead of print, 2021 Jan 19]. Clin Neurol Neurosurg 202:106503

33 Steffen R, Lautenschlager S, Fehr J (2020) Travel restrictions and lockdown during the COVID-19 pandemic-impact on notified infectious diseases in Switzerland. J Travel Med 27(8):taaa180. https://doi.org/10.1093/jtm/taaa180
34. Chan CP, Wong NS, Leung CC, Lee SS (2020) Positive impact of measures against COVID-19 on reducing influenza in the Northern Hemisphere. J Travel Med. 27(8):taaa087. https://doi.org/10. 1093/jtm/taaa087

Publisher's note Springer Nature remains neutral with regard to jurisdictional claims in published maps and institutional affiliations. 